\title{
Concordancia de monitores de temperatura para el postoperatorio inmediato. Cohorte observacional
}

\author{
Paz V. ${ }^{1}$, Vásquez J. ${ }^{1}$, San Martín P. ${ }^{1}$, Torres D. ${ }^{1,2}$ \\ 1 Universidad de Los Andes, Santiago, Chile. \\ 2 Clínica Santa María, Santiago, Chile.
}

Introducción: La monitorización de temperatura central en el intraoperatorio está estandarizada mediante monitores invasivos o de flujo de calor cero ( $\mathrm{FC} 0$ ), pero en recuperación los métodos invasivos son incómodos para los pacientes.

El objetivo de este estudio fue evaluar la concordancia entre el monitor de FC0 (Gold estándar) con dos termómetros no invasivos en el postoperatorio inmediato.

Métodos: Diseño: Cohorte observacional. Luego de aprobación por IRB, se incluyeron cincuenta pacientes adultos que ingresaron a la unidad de recuperación postanestésica después de haber sido sometidos a anestesia general. Después de la monitorización inicial, a todos los pacientes se les puso el monitor de FC0 (SpotOn, 3M, USA) y cuando se estabilizó la lectura, se realizó la medición simultánea con los dos métodos no invasivos: un monitor sublingual electrónico (SLE) (FILAC 3000 AD Electronic thermometer, Covidien, USA) y un dispositivo infrarrojo (IRT) (DT-8806C IR Thermometer, China). Todos los monitores fueron debidamente calibrados y se utilizaron siguiendo las indicaciones del fabricante. Las mediciones se realizaron con cada dispositivo, cada 10 minutos, hasta completar 90 minutos de monitorización. Estadística: Se describen las variables basales de los pacientes y se calculó el coeficiente de correlación intraclase (ICC) para cada instrumento comparado con el gold estándar y diferencia de promedios con ANOVA pareado (Bonferroni-Scheffe) (Stata 15. 2017. StataCorp LLC). La correlación se consideró estadísticamente significativa con $\mathrm{p}<0.05$ y se consideró significación clínica con diferencia $<0,5^{\circ} \mathrm{C}$.

Resultados: Datos de la cohorte en Tabla 1. Concordancia ICC entre FC0/SBE y FC0/IRT fue buena/moderada en todas las mediciones (Gráfico 1). Ambos termómetros mostraron una diferencia promedio menor de $0,5{ }^{\circ} \mathrm{C}$ comparado con FC0 en cualquiera de las mediciones, por lo que la diferencia no es clínicamente relevante (Gráfico 2).

Conclusiones: Tanto el monitor SBE como el IRT son precisos en la monitorización de temperatura en el postoperatorio inmediato, con una mejor concordancia en el caso de SBE. Cualquiera de los tres monitores se puede utilizar indistintamente en la unidad de recuperación.

https://doi.org/10.25237/congresoclasa2019.96 